\title{
Leibniz. Del panlogicismo y la metafísica del cálculo o de cómo imaginar una harmonia praestabilita
}

\section{Leibniz. Of Panlogicismus and the Metaphysics of Calculus or How to Imagine a Harmonia Praestabilita}

\author{
Jesús Carlos Hernández Moreno \\ Universidad Nacional Autónoma de México
}

\section{Resumen:}

La filosofía de Leibniz es considerada por los historiadores de la filosofía de diferentes y hasta opuestas maneras. En efecto, hay quienes consideran a Leibniz como "el gran espíritu universal de la modernidad" (Otto Saame) o como el filósofo pasado "de quien resultan hoy vigentes mayor número de tesis" (José Ortega y Gasset), pero también quienes consideran que su importancia no es "tan enorme que no se pueda concebir la historia del espíritu humano sin ella" (H. Glockner). Sin caer en estas descripciones lo que llevo a cabo en el artículo es un ensayo de comprensión de la filosofía de Leibniz según la cual ésta sería un panlogicismo en el que, a través de una metafísica del cálculo, se imagina una harmonia praestabilita.

Palabras clave: Leibniz, armonía preestablecida, panlogicismo

\section{Abstract:}

Leibniz's philosophy is considered by historians of philosophy in different and even opposite ways. In fact, there are those who consider Leibniz as "the great universal spirit of modernity" (Otto Saame) or as the past philosopher "of whom the greatest number of theses are in force today" (José Ortega y Gasset), but also those who consider that its importance is not "so enormous that the history of the human spirit cannot be conceived without it" (H. Glockner). Without falling into these descriptions, "this article presents an attempt to understand Leibniz's philosophy 
according to which this would be a panlogicism in which, through a metaphysics of calculus, a harmonia praestabilita is imagined.

Keywords: Leibniz, pre-established harmony, panlogicism

Leibniz es llamado por Otto Saame "el gran espíritu universal de la modernidad" (Saame, 1987: 31). Y sería, sin duda, universal si nos atenemos al sentido de lo prolífico y lo polifacético que es en sus escritos, de los que se nos dice que son o filosóficos, o matemáticos, o físicos, o biológicos, o teológicos, o históricos, o jurídicos, etcétera. ${ }^{1}$ Leibniz recorrería así las distintas dimensiones del universo de la modernidad, alcanzando incluso temáticas que llamarán fuertemente la atención del pensamiento contemporáneo, a pesar de tener que ostentar este último como talante lo moderno, esto es, lo pretendidamente superador de modernidades no tan modernas.

En esta comprensión que divide, que se afana en señalar su especificidad en detrimento de la generalidad, sería como si el espíritu de Leibniz, esa mónada tan capaz de dar razón, se desplazara cómodamente multiplicando no sólo la sustancia en sustancias, ${ }^{2}$ sino también la ciencia en ciencias. ¿¿Sería empero atinado pensar a Leibniz como un pensador ocurrente que ahora inaugura ciencias por aquí y por allá, sin que se deje claro por qué la ciencia puede ser entendida separada, esto es, como ciencias? Es más, ¿podría haber ciencias sin que hubiere propiamente comunicación entre ellas, esto es, cientificidad que las fundara legítima y legalmente como tales ciencias, y sin que simplemente dicha cientificidad fuera propia y claramente la ciencia? Además, ¿qué significaría que el pensamiento de Leibniz pudiera tener tantas parcelas que le permitiera triunfar en algunas de ellas, aunque en otras no pudiera de ninguna manera hacerlo? ¿Qué se pretende al señalar el saber dividido y justificarse para ello, por ejemplo, en el modo en el que se predica que el trabajo se dividió para entrar en una nueva era de prosperidad económico-social para los seres humanos? Esto último, dividir el saber en saberes como mecanismo de progreso del mismo es algo que entusiasmó tanto, entre otros, a ese leibniziano

${ }^{1}$ Escritos que, al día de hoy y a pesar de los esfuerzos concentrados en la Edición de la Academia, todavía no conforman integralmente una edición completa, lo que vuelve más o menos inseguros no pocos de los trabajos dedicados exclusivamente al pensamiento de Leibniz, aunque no por ello despreciables. Ya José Ortega y Gasset decía que "la excesiva riqueza de su pensamiento [...] le impidió dar nunca expresión sistemática a sus ideas hipersistemáticas” (Ortega y Gasset, 1962: 88).

${ }^{2}$ Para Leibniz, como veremos, la sustancia es plural y es llamada mónada; y las mónadas son espirituales y distintas entre sí. 
crítico que fue Immanuel Kant, a tal punto que en el "Prólogo" a su Grundlegung zur Metaphysik der Sitten lo supuso como modelo para la filosofía, acusando la falta del mismo como "la mayor barbarie" para los oficios. ${ }^{3}$ Aunque, por el contrario, René Descartes, por ejemplo, a quien se le ha querido señalar habitualmente, no sin sembrar sospechas, como precursor de Leibniz, estimaba dicha diversidad de ciencias como una falsificación de la ciencia una (mathesis universalis), aplicable, eso sí, como infinitas dimensiones de cosas. ${ }^{4}$

¿La ciencia es una, es múltiple o es una oscilación entre unidad y multiplicidad? Esto, por cierto, es algo que ha ocupado a los filósofos desde los antiguos griegos aunque no propiamente planteado respecto a la naturaleza de la ciencia sino de todas las cosas y en general se puede entender como la posibilidad de la multiplicidad de principios para atender la comprensión de su constitución, o la posibilidad de la unicidad de un principio para hacerlo, o, subsumiendo ambas posibilidades, de un eterno vaivén de una posibilidad a otra, como, por ejemplo, pensaría Empédocles de Agrigento. Las apuestas por resolver esta tensión en favor de alguna de las posibilidades al respecto atraviesan analógicamente las múltiples dimensiones de predicación de las cosas que científicamente se han dado lugar, desde lo ontológico hasta lo político. En lo que respecta a las apuestas políticas tendría lugar esta tensión, por ejemplo, en las que hablan de habitar como una iglesia y/o también habitar como un imperio comunicador de pueblos frente a los que hablan de habitar afirmando una pluralidad de iglesias y/o también de naciones que, aún en su posible articulación confederada, no obstante se reclaman desde su distinción nacionalista. ${ }^{5}$

Yo planteo la cuestión aquí sólo desde un afán de comprensión de lo epistemológico. Y de esa manera ya la ensayaba, por ejemplo, Platón al hacer que Sócrates censurara a Teetetes cuando en su respuesta a la pregunta por la ciencia este último atinó sólo a seńalar la multiplicidad de las ciencias y no a la cientificidad en ellas ${ }^{6}$;

\footnotetext{
3 "Todas las industrias, oficios y artes han ganado mucho con la división del trabajo [...]. Donde las labores no están así diferenciadas y divididas, donde cada hombre es un artífice universal, ahí yacen aun en la mayor barbarie" (Kant, 1999: v-vi).

${ }^{4}$ Véase de Descartes Regulae ad directionem ingenii, I, IV y XIV; y también la primera y la segunda partes del Discours de la méthode.

${ }^{5}$ Llama la atención que la apuesta divisoria de las ciencias en la época de Leibniz tenga algún íntimo paralelismo con lo que al respecto sucedió en la religión, que dividió cristianismos, o en la política, que dividió imperios. $\mathrm{Al}$ respecto, vale la pena atender las Cartas sobre tolerancia y los programas de "leyendas negras" y de construcción de nacionalidades, con sus símbolos y sus pedagogías. Así como la encíclica Pascendi domini gregis de Pío X en la que acusa como artimańa de los modernistas el presentar su pensamiento en fragmentos aunque por lo general tengan claro lo apostado en ellos desde el principio. Sobre estos temas arrojan luz, por ejemplo, los libros La religión de Descartes de Juan Carlos Moreno Romo e Imperofobia y leyenda negra de Elvira Roca.

${ }^{6}$ Platón, Teeteto, $146 \mathrm{c}$ y ss.
} 
y también lo ensayaba ya Aristóteles cuando, por ejemplo, pensaba en la sofía como la ciencia más ciencia o la ciencia en sentido estricto, ${ }^{7}$ esto es, la ciencia primera, y, se podría decir que, por tanto, de primera, ciencia que, de hallarse, dejaría a las otras en el estatus de ciencias más bien de segunda. ${ }^{8}$ Y se podría decir que ya en la llamada modernidad es también lo que atienden destacadamente tanto el denominado "caballero de la luz", René Descartes, como el autodenominado "quijote de la filosofía”, Arthur Schopenhauer: en el primero, según ya se ha señalado, es el método (mathesis universalis) la ciencia misma, ${ }^{9}$ mientras que para el segundo es la mundosofía (Weltweissheit) entendida como un único pensamiento demorado en consideraciones suficientemente atentas y correlativas del mundo. ${ }^{10}$ Pero no me interesa aquí abordar dicha cuestión sobre la naturaleza de la ciencia en general, sino sólo en el afán de comprender el pensamiento de Leibniz. ¿Qué pasa pues respecto a esta cuestión filosófica acerca de la naturaleza de la ciencia en la apuesta de pensamiento de Leibniz, que es la que concentradamente atiendo en lo que aquí ensayo? ¿Es Leibniz pensador de una unicidad en la cientificidad o de una pluralidad en ella?

Si el pensamiento de Leibniz es visto no como pensamiento en la modernidad sino como un objeto de estudio de la modernidad y la modernidad es entendida no en el sentido que apuesta el pensamiento filosófico de Descartes sino en el que lo hace el pensamiento cientificista y discriminatorio de la Ilustración, ${ }^{11}$ entonces dicho pensamiento no podría sino quedar dividido y divisible tendencialmente como ad infinitum. Así, los trabajos de Leibniz quedarían repartidos en múltiples ciencias y, en la difusión de lo que se publicita a favor de esta afirmación acerca de la naturaleza de la ciencia, se podría decir que Leibniz aunque ahora fuera ya inútil en física, no necesariamente lo sería en matemáticas, y aunque su lógica fuera insuficiente, podría de todos modos subsistir por su metafísica, quedando por tanto,

${ }^{7}$ Ibid., 982 b 1 (traducción de Valentín García Yebra). Tomás Calvo Martínez traduce "la ciencia en grado sumo".

${ }^{8}$ Aristóteles, Metafísica.

${ }^{9}$ Véanse las referencias de nuestra nota al pie de página número 5.

${ }^{10}$ Véase el prólogo a la primera edición de Die Welt als Wille und Vorstellung y el capítulo 17 de Ergänzungen a dicha obra.

${ }^{11}$ La Ilustración es una moda política y social que discriminará hasta crear cosas como el racismo, que predicará científico y, por tanto, verdadero, de donde concluiría que es moralmente bueno a pesar de todo el daño que ha desencadenado como mal para los que lo han tenido que padecer, que de alguna manera hemos sido todos (Mosse, 1990). Sobre la diferencia de racionalismos modernos o de la Ilustración con un proyecto no cartesiano, véase el texto de Juan Carlos Moreno Romo, "El trasfondo espiritual de nuestros racionalismos. O sobre el preciso punto en el que difieren la modernidad más bien luterana y la genuinamente cartesiana”. 
al menos, "como alguien de quien todo el mundo puede aprovechar algo" (Martínez Marzoa, 1991: 11). Esto último es lo que Felipe Martínez Marzoa denuncia que se ha hecho con el pensamiento de Leibniz en ese afán de dividir sin reparar en que "cada hallazgo matemático de Leibniz es, en Leibniz, un paso determinado del proyecto filosófico y tiene el sentido que ese proyecto le da" (1991). Martínez Marzoa deja ver que la "universalidad" de Leibniz podría considerarse de otra manera que bajo la lectura que simplemente la fragmenta. Para él está claro que Leibniz no ha "sido bien tratado por la historia" (1991).

Aunque el pensamiento de Leibniz fuera visto a través de aquella perspectiva que lo fractura, Leibniz, en cada trozo de la ciencia y en el conjunto de éstos, aparecería de todos modos con un tamańo mayor que el de no pocos de los más afamados genios de los pensamientos de la modernidad. En comparación con los otros modernos, en lo moderno, que es destacadamente cuantitativo, Leibniz difícilmente podría hallar par. A eso es a lo que apunta, por ejemplo, José Ortega y Gasset cuando en La idea de principio en Leibniz y la evolución de la teoría deductiva dibuja, en lo moderno, el tamańo de Leibniz que, por ejemplo, a pesar de la publicidad que ha gozado Isaac Newton, sir para los ingleses - y no pocos espírituscolonia-, no tiene frente a él comparación. Escribió Ortega y Gasset:

Leibniz vivió en combate permanente contra Newton. Esta polémica ha sido una de las más excelsas gigantomaquias que en el planeta se han dado, y es una vergüenza que aquel egregio pugilato no haya sido aún contado de manera condigna ni en su lado doctrinal ni en su lado "humano". Este último es también sobremanera interesante, porque en él vemos que Newton es, de los dos, quien ha tenido siempre "buena Prensa", mientras que Leibniz la ha tenido siempre mala, empezando por el genio del periodismo: Voltaire. El caso es tanto más escandaloso cuanto que en aquella polémica, según ahora vemos, era Leibniz quien "llevaba la razón” sobre la mayor parte de las discrepancias, y llevaba la razón en un grado que casi parece, repito, sobrehumano. Leibniz anticipa con una clarividencia que produce escalofrío lo que en nuestro tiempo ha llegado a ser tanto la pura matemática más reciente como la más reciente física. Porque es preciso hacer constar que es Leibniz, de todos los filósofos pasados, aquel de quien resultan hoy vigentes mayor número de tesis. (Ortega y Gasset, 1962: 88)

Ortega y Gasset estaba convencido de que Leibniz había enriquecido sobremanera la ciencia con ciencias. Y, sin duda, frente a su pensamiento tan rico, en tanto que amplio, fácilmente se podría sentir la inclinación de decir con él que Leibniz "hoy mismo nos produce una impresión desazonadora, como si estuviése- 
mos en presencia de una hiperlucidez extrahumana, de un alma sin cesar fosforescente, que viajando en carroza creaba ciencias enteras" (Ortega y Gasset, 1962: 88).

Pero, insisto, ¿ampliar la ciencia quiere decir también ampliarla en ciencias? Y, además, si con el pensamiento rico, por amplio, de Leibniz, se amplía la ciencia, ¿querrá esto decir también que, con ella, la ciencia, se ha enriquecido igualmente la filosofía?, ¿o acaso lo ha hecho en detrimento de la filosofía por ser su metafísica lo más desestimado y hasta burlado de su pensamiento ${ }^{12}$ ¿Pero es que bajo qué tipo de malentendido se quiere separar de suyo ciencia y filosofía haciéndolas entrar así en variopintas dependencias que les instituye y hasta les opone?, ¿qué pretensiones auténticamente filosóficas tiene éste?

Sea como fuere, Leibniz es un pensador que, aun cuando se le quisiera ver como asistemático y fragmentario por la diversidad de sus escritos, puede no obstante decir: "[...] en mi sistema [...] hay en todo una perfecta conexión" (citado en Saame, 1987: 33) o, según escribe en otra parte, "todo está conectado" (1987).

Y, en efecto, su pensamiento "trata de una sola cosa” (Martínez Marzoa, 1991: 13) y "aunque muy ramificado, [...] es, en realidad, unitario" (Saame, 1987: 34), como también observa Otto Saame, pues tiene como eje inquebrantable el principio de razón, principio a la luz del cual cobraría sentido en este pensamiento todo otro principio, en específico, el principio de identidad o de contradicción y el principio de razón suficiente. Así, aunque Ortega y Gasset lo llame "el hombre de los principios"13 (Ortega y Gasset, 1962: 64, 89) por distinguir en su pensamiento

\footnotetext{
${ }^{12}$ Esto puede verse claramente ya desde 1753 cuando la Real Academia Prusiana de Ciencias de Berlín, que Leibniz fundara en 1700, es presidida entonces por Pierre-Louis Moreau de Maupertuis, quien al tocarle el turno a la "Clase filosófica" de convocar a un concurso, indirectamente se propone atacar a Leibniz: "Se exige un examen del sistema de Pope contenido en la proposición "Todo está bien". Se trata: (1) de determinar el verdadero sentido de esta proposición según las hipótesis de su autor; (2) de comparar el sistema de Pope con el sistema del optimismo, o la elección de lo mejor, para indicar exactamente las semejanzas y diferencias entre aquellos sistemas, y finalmente (3) de presentar las razones por las cuales se debe aceptar o destruir este sistema" (Caro, 2005: 48). El ridículo fue tal que escandalizó a los intelectuales alemanes, a tal punto que en 1755 responden satírica y anónimamente a esta convocatoria Lessing y Moses Mendelssohn con un escrito titulado Pope: ;un metafísico!. A los ataques de Maupertuis sigue la burla que Voltaire hace de Leibniz en 1759 en su cuento Candido, o el optimismo, de la que ya algo había en su Poema sobre el desastre de Lisboa o examen de este axioma: todo está bien (enero de 1756). También hay que señalar al "leibniciano" Kant, que, bajo el mote de "dogmática", arremetió "críticamente" contra la metafísica de Leibniz constituyendo con ello prácticamente un "dogma" de los estudios kantianos. En fin, incluso Arthur Schopenhauer encuentra a Leibniz como un caso filosófico especial para "ensañarse" con él.

13 "Leibniz nos aparece, entre los filósofos, como siendo por excelencia el "hombre de los principios" (Ortega y Gasset, 1962: 89). Ortega y Gasset lista diez principios: "el principio de los principios", "principio de identidad", "principio de contradicción", "principio de la razón suficiente", "principio de la uniformidad o principio de Arlequín", "principio de la identidad de los indiscernibles o principio de la
} 
diez principios, hay que insistir también aquí, como con la pregunta sobre la unicidad o la pluralidad de la ciencia, que, no obstante dicha pluralidad de principios, todos éstos están también interconectados y gobernados por el principio de los principios, el principio de razón.

Cada uno de estos dos principios que hemos destacado a la luz del principio de razón, el principio de identidad o de contradicción y el principio de razón suficiente, son principios que rigen distintos órdenes, pues, al establecerse ya un principio, debe poderse desplegar desde él un orden (o mundo) en el cual él rija como tal, esto es, como principio, pero sin que por esto se anule la subordinación en la que se encuentran ambos principios y sus órdenes respecto del orden mayor desplegado desde el principio de razón en el que éstos tienen lugar. Así,

El principio de "identidad o contradicción" constituye el "gran fundamento de la matemática” (le grand fondement des mathématiques). De él se pueden deducir todos los principios de la aritmética y de la geometría. [Mientras que,] por el contrario, el principio de razón suficiente impera, según Leibniz, en la "mecánica”, en "física, teología natural”, "metafísica” y en la "moral”. (Saame, 1987: 41)

En efecto, Leibniz, aparentemente fiel al dualismo instaurado por Descartes, ${ }^{14}$ establece dos ámbitos de validez distintos para cada principio relativamente absoluto, como da cuenta de ello en la quinta carta que le escribe a Clarke:

En mi Teodicea he mostrado suficientemente que esta necesidad moral es satisfactoria, conforme con la perfección Divina, conforme con el gran principio de las existencias, que es el de la necesidad de una razón suficiente, mientras que la necesidad absoluta y metafísica depende del otro gran principio de nuestro razonamiento, que es el de las esencias, es decir, el de la identidad o la contradicción, puesto

diferenciación", "principio de continuidad", "principio de lo mejor o de la conveniencia", "principio del equilibrio o ley de justicia" y "principio del mínimo esfuerzo o de las formas óptimas". De estos diez principios, cabría señalar que, para entender la filosofía de Leibniz, los más importantes son los primeros cuatro, los cuales constituyen propiamente sólo tres, pues el segundo y el tercero en realidad son "la meme chose". De estos tres, "el principio de los principios" es el "principio de razón", no el de "razón suficiente", que tiene un "dominio menor", pues sólo es válido para la "existencia”, esto es, "para el ámbito de las 'verdades de hecho"' (Saame, 1987: 40), bajo el que quedan relacionados los otros dos principios.

${ }^{14}$ Dualismo del que en otro lugar he intentado mostrar que sólo "epistemológicamente" es en Descartes tal, véase Hernández, 2017. Respecto a este tema acerca del dualismo ontológico apostado por Leibniz y por Lutero, destacan los trabajos de Juan Carlos Moreno Romo, "De Elisabeth a Leibniz. ¿Está el dualismo luterano en el trasfondo del dualismo cartesiano?” en La religión de Descartes; de Jean-Luc Nancy, Ego sum, y de Jean-Luc Marion, Sobre la ontología gris de Descartes. 
que lo que es absolutamente necesario es lo únicamente posible entre las alternativas y su contrario implica contradicción. (Leibniz, 1890: 390-391, párrafo 10) ${ }^{15}$

El principio de razón se expresa en dos órdenes distintos en tanto que principio de identidad o no contradicción y también en tanto que principio de razón suficiente, por eso es que ya desde él quedan preestablecidos los armoniosos comportamientos que dichos órdenes expresan.

Respecto a las llamadas ciencias (aritmética, geometría, mecánica, física, teología natural, metafísica y moral, y las que de éstas pudieran derivarse), éstas no son sino expresiones de estos dos principios y estos últimos no son sino formas, según este pensamiento, distintas ${ }^{16}$ del principio de los principios, esto es, del principio de razón. El principio de razón no dice otra cosa sino que "nada sucede sin una razón" (rien n'arrive sans raison), lo que en Leibniz quiere decir no sólo que todo sea explicable por un mecanismo excogitado que permite inventar órdenes, como sería metódicamente en lo finito descrito por Descartes, sino que, y en esto se encuentra, según vemos, la confusión u "ontologización de lo epistemológico" (Blanco Pérez, 2005: 257) en Leibniz, todo está fundamentado, pues “omne praedicatum inest subiecto". Sobre esto último escribe Leibniz en su Discours de Métaphysique

Es muy cierto que, cuando varios predicados se atribuyen a un mismo sujeto, y este sujeto no se atribuye a ningún otro, se le llama sustancia individual, pero esto no es bastante y una tal explicación no es más que nominal. Es preciso, pues, examinar qué es eso de ser atribuido verdaderamente a un cierto sujeto. Ahora bien, consta que toda predicación verdadera tiene algún fundamento en la naturaleza de las cosas, y cuando una proposición no es idéntica, es decir, cuando el predicado no está comprendido expresamente en el sujeto, hace falta que esté allí contenido virtualmente, y a esto le llaman los filósofos in-esse, diciendo que el predicado está en el sujeto. Así, es preciso que el término del sujeto encierre siempre el del predicado, de suerte que el que entendiese perfectamente la noción del sujeto, juzgaría también que el predicado le pertenece. (Leibniz, 1880: VIII, 432-433)

15 "Et j'ay assés montré dans ma Theodicée, que cette necessité murale est heureuse, conforme à la perfection Divine, conforme au grand principe des existences, qui est celuy du besoin d'une raison suffisante; au lieu que la necessité absolue et metaphysique depend de l'autre grand principe de nos raisonnemens, qui est celuy des essences, c'est à dire celuy de l'identité ou de la contradiction: car ce qui est absolument necessaire, est seul possible entre les partis, et son contraire implique contradiction."

${ }^{16}$ Esto es, "que se distinguen en" no "que se distinguen de". 
La decisión de Leibniz de una filosofía que parta de principios a la luz de la noción moderna de racionalidad, entendida ésta como una realidad espiritual ordenadora de pensamientos, lo confinará en los argumentos desde los cuales, como él mismo concluía, no es posible alcanzar una realidad corpórea: "En consecuencia, ningún argumento puede demostrar absolutamente la existencia de los cuerpos (Itaque nullo argumento absolute demonstrari potest, dari corpora)" (Leibniz, 1880: 320), escribe Leibniz. De aquí que toda posibilidad de pensar al margen de la lógica se desvanezca en su pensamiento, pues respecto al problema acerca del conocimiento de la realidad externa, que se halla inmerso destacadamente en la discusión del horizonte epistemológico de Descartes, Leibniz pone a hablar también a la lógica, que, así, impera sobre lo epistemológico y lo ontológico. Tal lógica sería en consecuencia la filosofía, la ciencia y la vida para Leibniz. Por esto es que su filosofía ha sido llamada certeramente "panlogicismo", pues en ella, el universo mismo, si está en lo correcto Aron Gurwitsch, no era sino ex-presión de la lógica (Gurwitsch, 1974: 4). ${ }^{17}$

Leibniz, apostado en ese horizonte moderno del yo en el que, con Descartes, el entendimiento y sus axiomas eran capaces de ordenar e inventar posiblemente infinidad de dimensiones en las cosas (subjectis) (Descartes, 1913: 448), negará, contra Newton y Clarke - anclados también a dicho horizonte moderno del yo $^{18}$ — , la sustancialidad, esto es, la realidad absoluta del espacio y del tiempo, eso que para Kant serán las subjetivas condiciones de posibilidad de lo sensible. Leibniz hará de ellos órdenes de relación, donde los sujetos, sin apertura a una

17 "La concepción del universo como encarnación de la lógica require un sujeto de la lógica, un espíritu y operaciones espirituales, cuyo correlato constituye el universo (Die Auffassung des Universums als Inkarnation von Logik erfordert ein Subjekt der Logik, einen Geist und geistige Operationen, deren Korrelat das Universum bildet)".

${ }^{18}$ La epistemología excogitada por Descartes tiene, según hemos visto, un dualismo como base en el que hay dos (pseudo)sustancias que, gracias a Dios tienen realitas: res cogitans y res extensa. Ni Leibniz ni Newton y Clarke (y tampoco Locke ni Malebranche ni Spinoza) pondrán en duda el supuesto "Dios”. Tampoco se distinguirán en sus esfuerzos por superar $e l$ dualismo "cartesiano" y en hacerlo mediante una confusión de lo epistemológico y lo ontológico (Hernández Moreno, 2011: 41-48) y, para ello, recurrir al presupuesto de racionalidad a su modo, mecanizante o dinamizante, que se acusa a Descartes de instaurar. Sin embargo, sus diferencias radicarán en que unos y otros lo "superarán" apostando "ontológicamente" ya a una o ya a la otra parte de dicho dualismo. Sobre esto es que la historia de la filosofía pudo simplificar (- ¿qué más podría hacer la historia?-) estos pensamientos en racionalistas y empiristas, siendo los primeros los que inventaban mundos basados en la comprensión que del cogito tenían, mientras que los segundos lo hacían respecto de la res extensa. 
exterioridad, ${ }^{19}$ serán sujetos de una ars combinatoria en la que no serán alterados y, sin embargo, fundarán toda posible relación, permitiendo así que cálculo y ser sean la expresión de "una sola cosa" (Martínez Marzoa, 1991: 13) que, dicho en una expresión vacía, ${ }^{20}$ sería "lo que es". ${ }^{21}$

En esta filosofía hay, lógicamente, realidad de los sujetos, pues éstos son las unidades que sostienen sus predicados. Mas, ¿qué es para Leibniz un sujeto? La respuesta no encuentra mejor lugar en la obra de Leibniz que al inicio de la Monadologie (Principia philosophiae seu thesis in gratiam principis Eugenii conscriptae), que es la obra en la que esquematiza sistemáticamente su panlogicismo como un pluralismo sustancialista preestablecidamente armonioso.

El sujeto, en sentido estricto, es nombrado por Leibniz "mónada”. Sin embargo, no se trata, como podría derivarse de las afirmaciones en Descartes, de, propiamente, una sola sustancia, sino, por el contrario, de una pluralidad de sustancias. En efecto, la pretensión leibniciana de superar el abismo entre la res extensa y la res cogitans le confinará en un atomismo inmaterialista (Leibniz, 1885: 1, 3, 12) dado que la materia, que es vista geométricamente, es divisible infinitamente y, por tanto, al seguir siendo compuesta, pues tiene partes, no encuentra propiamente sustancialidad, ya que ésta, la materia, está en relación de dependencia con lo que la compone. Por eso, estrictamente, para Leibniz, no existe nada material ni extenso (1885), sino sólo apariencia de tal, pues "lo compuesto no es otra cosa que cúmulo o aggregatum de simples" (1885). ${ }^{22}$ Por tanto, sólo existen propiamente las mónadas, esto es, dichas "substances simples" (1885).

De la simplicidad de estas sustancias Leibniz excluirá el “(inter)cambio” (Leibniz, 1885: 7), por lo que, escribe, "no hay ninguna manera concebible por la cual una sustancia simple pudiera perecer naturalmente" $(1885),{ }^{23}$ pero, por la misma razón, tampoco "pudiera comenzar naturalmente" (1885). ${ }^{24}$

Sin embargo, al afirmar la (pseudo)idea de creación, que implica la intervención de un Creador de otra naturaleza que la de la creatura, Leibniz establecerá una diferencia ontológica entre Dios y todas las demás substancias simples, todas mó-

19 “Les Monades n'ont point de fenêtres, par lesquelles quelque chose y puisse entrer ou sortir." (Leibniz, 1885: 7)

20 ¿De qué otra manera no sustancializante podría decirse "que-es" o "que sin qué”?

${ }^{21}$ Aquello que en el Libro Z de los libros de Metafísica Aristóteles escribiera que es lo que antes, ahora y siempre se pregunta el filósofo, y que él pone en término de ousía.

22 "[L]e composé n'est autre chose qu'un amas ou aggregatum des simples" (Leibniz, 1885: 2).

23 "II]l n'y a aucune manière concevable par laquelle une substance simple puisse périr naturellement" (Leibniz, 1885: 4).

24 “puisse commencer naturellement” (Leibniz, 1885: 5). 
nadas (incluido Dios), que hará a las sustancias de naturaleza distinta a la de Dios susceptibles de cambio en su naturaleza (Leibniz, 1885: 10), ${ }^{25}$ esto es, de ser o no-ser, a tal punto que "las Mónadas [,excepto la mónada Dios,] no pueden comenzar ni terminar de repente, es decir, ellas no pueden comenzar más que por creación y terminar más que por aniquilación [o reducción a nada ${ }^{26}$ ” $(1885) .{ }^{27}$ De aquí que ninguna mónada pueda ser alterada "en su interior por ninguna otra creatura” (1885), ${ }^{28}$ pues, según veíamos más arriba, ellas no se comunican debido a que "no tienen ventanas" (1885). ${ }^{29}$

Además, estas mónadas necesitan para ser, de acuerdo con Leibniz, de cualidades propias ${ }^{30}$ y diferentes entre ellas, ${ }^{31}$ si no, "las Mónadas [...] serían indistinguibles las unas de las otras [...] y un estado de cosas sería indiscernible de otro" (Leibniz, 1885: 8, 9), ${ }^{32}$ lo que no permitiría explicar, por tanto, las diferencias de la (pseudo)realidad fenoménica. En consecuencia, escribe el también cortesano, "los cambios naturales de las Mónadas vienen de un principio interno" (1885). ${ }^{33}$ Pero agrega que, además del principio de cambio, debe haber "un detalle de lo que cambia, que, por así decirlo, haga la especificación y la variedad de las sustancias simples" (1885), ${ }^{34}$ el cual "debe envolver una multitud en la unidad o en lo simple" $(1885)^{35}$ y que no es más que un estado pasajero (état passager) identificado con la percepción (Perception) distinto de la apercepción o de la conciencia (1885). ${ }^{36} \mathrm{La}$ "acción del principio interno", que es la que realiza el cambio o el paso de una percepción a otra, es llamada aquí "Apetición" (Appétition) (1885). Sin embargo, según confiesa Leibniz, "la Percepción y lo que de ella depende es inexplicable por razones mecánicas"

25 “[T] out être créé est sujet au changement, et par conséquent la Monade créée aussi”.

${ }^{26}$ En el latín "annichilare” es una forma alterada del latín tardío "annihilare", que quiere decir "reducir a nada".

27 “[L]es Monades ne sauraient commencer, ni finir, que tout d'un coup, c'est-à-dire, elles ne sauraient commencer que par création et finir que par annihilation” (Leibniz, 1885: 8).

28 "[D]ans son intérieur par quelque autre créature" (Leibniz, 1885: 7).

29 "[Elles] n'ont point de fenêtres" (Leibniz, 1885: 7).

30 "Cependant il faut que les Monades aient quelques qualités, autrement ce ne seraient pas même des êtres” (Leibniz, 1885: 8).

31 "Il faut même, que chaque Monade soit différente de chaque autre" (Leibniz, 1885: 9).

32 "[L]es Monades [...] seraient indistinguables l'une de l'autre [...] et un état des choses seait indiscernable de l'autre" (Leibniz, 1885: 8).

33 "[L]es changement naturels des Monades viennent d'un principe interne" (Leibniz, 1885: 11).

34 " $[U] n$ détail de ce qui change, qui fasse pour ainsi dire la spécification et la variété des substances simples" (Leibniz, 1885: 12).

35 "[D]oit envelopper une multitude dans l'unité ou dans le simple" (Leibniz, 1885: 13).

${ }^{36}$ Esta distinción es, según Leibniz, en lo que se han equivocado los cartesianos, pues no tuvieron "en cuenta para nada aquellas percepciones de las que uno no se da cuenta" (Leibniz, 1885: 13). 
(1885). ${ }^{37} \mathrm{Y}$, concluye, "sólo en esto es en lo que pueden consistir todas las acciones internas de las substancias simples" (1885). ${ }^{38}$

Estas sustancias, distinguibles entre ellas, permiten ser clasificadas por Leibniz en tres tipos distintos, tomando como criterio para ello lo que realiza en ellas "las consecuciones de sus percepciones" (les consécutions de leurs perceptions) (Leibniz, 1885: 28): 1) "Mónadas desnudas" (Monades toutes nues), las cuales sólo tienen percepción sin conciencia (1885); 2) mónadas cuya percepción es acompañada por memoria y conciencia, como en el caso de los animales (1885); y 3) mónadas que, además de conciencia y memoria, tienen Razón (1885). ${ }^{39}$

La Razón, por medio de la cual son conocidas las “verdades necesarias y eternas", (se) funda, como hemos visto ya, en los principios de contradicción o de identidad y de razón suficiente (Leibniz, 1885: 31-32). El segundo rebasa los límites de las verdades lógico-matemáticas y alcanza incluso a Dios, que sería el límite supremo, "la dernière raison des choses" (1885). Leibniz también establece, basado en estos dos principios que parecen regir distintos órdenes de la verdad, la división de las verdades en verdades de hecho y verdades de razón (1885). ${ }^{40}$ El rasgo característico de cada una de estas clases de verdad sería, para las verdades de razón, la necesidad, y, para las verdades de hecho, la contingencia. Las primeras serían gobernadas por el principio de identidad y su razón puede ser hallada por análisis hasta llegar a las "nociones primitivas" (principes primitifs) (1885), indemostrables pero innegables por dicho principio de identidad o de contradicción, porque, según Leibniz, estas Énonciations identiques no permiten su opuesto sin expresar una evidente contradicción (1885). El ejemplo paradigmático de éstas se encuentra en el quehacer de los matemáticos, que muestran la reducción de sus teoremas a definiciones (Définitions), axiomas (Axiomes) y proposiciones (Demandes) (1885). Las segundas, esto es, las vérités de Fait están caracterizadas por la contingencia (1885), la cual, sin embargo, no es, según Leibniz, ninguna limitante para el poder de la Razón, pues, al ser, aunque esto sea fuera de la necesidad y sólo en la posibilidad, están obligadas a responder al principio de razón suficiente, que las alcanza y las gobierna revistiendo la posibilidad de necesidad, esto es, mostrándolas igualmente

\footnotetext{
37 "On est obligé d'ailleurs de confesser que la Perception et ce qui en dépend, est inexplicable par des raisons mécaniques, c'est-à-dire par les figures et par les mouvements” (Leibniz, 1885: 17).

38 "C'est en cela seul aussi que peuvent consister toutes les Actions internes des substances simples" (Leibniz, 1885: 17).

39 "Mais la connaissance des vérités nécessaires et éternelles est ce qui nous distingue des simples animaux et nous fait avoir la Raison et les sciences; en nous élevant à la connaissance de nous-mêmes et de Dieu. Et c'est ce qu'on appelle en nous Âme raisonnable, ou Esprit" (Leibniz, 1885: 29).

40 "Il y a aussi deux sortes de vérités, celles de Raisonnement et celle de Fait" (Leibniz, 1885: 33).
} 
lógicas. Sin embargo, esto sólo sería posible tras conceptualizarlas como partes componentes de "la sucesión de las cosas que se hallan repartidas por el universo de las criaturas" (1885), ${ }^{41}$ donde el concepto clave es el de sucesión (suite) (o series [séries]) (1885). Estos conceptos, como es sabido, son, junto al de límite, el corazón de ese instrumento moderno llamado cálculo infinitesimal $(1885)^{42}$ con el que se ha pretendido racionalizar el cambio; instrumento que, montado sobre la geometría de Descartes ${ }^{43}$ y dada la ontologización de lo epistemológico, ha sido con lo que se han tejido los saberes más pretenciosos que articulan la actual comprensión de mundo y que emblemáticamente se presentan como bandera y vanguardia de la civilización occidental, llamada por eso por Miguel de Unamuno como "civilización mecánica” (Unamuno, 1947). Leibniz establece por necesidad que la razón de dichas series no puede estar contenida en ellas, ${ }^{44}$ de donde concluye que ella no puede ser otra cosa que Dios: "Y así la razón última de las cosas debe estar en una substancia necesaria, en la cual el detalle de los cambios no esté sino eminentemente, como en su origen: y esto es lo que llamamos Dios" (Leibniz, 1885: 38). ${ }^{45} \mathrm{Y}$, dadas las características con las que se define a Dios, podrá concluir la necesidad de la existencia del mismo, por lo que todo debe estar asegurado "lógicamente" del modo descrito. En efecto, escribe Leibniz:

Por tanto, sólo Dios (o el Ser Necesario) tiene este privilegio: que es preciso que exista si es posible. Y como nada puede estorbar la posibilidad de lo que no implica límite alguno ni ninguna negación, y por consecuencia, ninguna contradicción, basta esto solo conocer la Existencia de Dios a priori. También lo hemos probado

41 “[L]a suite des choses répandues par l'univers des créatures” (Leibniz, 1885: 36).

${ }^{42}$ Llama la atención que sea igualmente atribuido a Leibniz y a Newton, aunque se llegó a acusar falsamente a Leibniz de plagiario, lo cual, por cierto, no es extrańo, pues como en la Ilustración la competencia de las naciones y sus "marcas registradas" de los pensamientos en la supuesta liberación de los mismos por la míticamente amítica Diosa Razón se volvió negocio nacional, por lo que era cosa frecuente la acusación de los plagios intelectuales.

43 "La fuente más completa sobre la vía que llevó a Leibniz al descubrimiento del Cálculo infinitesimal”, según comenta Javier Echeverría (Echeverría, 1995: 530), está contenida en De Quadratura Arithmetica Circuli, Ellypseos et Hyperbolae, cuius corollarium est Trigonometria sine Tabulis. Respecto de la geometría de Descartes basada en cuadrados, Leibniz “demuestra la posibilidad de reducir los rectángulos a triángulos [...e introduce] la proposición más importante al respecto [...: la de la transmutación (Teorema 7), en la cual Leibniz comienza a utilizar triángulos infinitesimales." (1995).

44 " ii]l faut que la raison suffisante ou dernière soit hors de la suite ou séries de ce détail des contingences, quelqu'infini qu'il pourrait être" (Leibniz, 1885: 37).

45 "Et c'est ainsi que la dernière raison des choses doit être dans une substance nécessaire, dans laquelle le détail des changements ne soit qu'éminemment, comme dans la source: et c'est ce que nous appelons Dieu" (Leibniz, 1885: 38). 
por la realidad de las verdades eternas. Pero también acabamos de probarlo a posteriori puesto que existen seres contingentes, que no tienen su razón última o suficiente más que en el ser necesario, el cual tiene la razón de su existencia en sí mismo. (Leibniz, 1885: 45)

Como se puede ver, hay en Leibniz la convicción de que Dios es racionalmente cognoscible y, además, de que posee, como para Descartes, ${ }^{46}$ entendimiento y voluntad, aunque aquí, a diferencia del modo en el que lo concebía Descartes, el entendimiento antecede ontológicamente a la voluntad (Leibniz, 1885: 46), generando capas ontológicas en Dios. Así, "en Dios hay la potencia [Puissance], que es el origen de todo, después el Conocimiento [Connaissance], que contiene el detalle, de las ideas, y, por último, la Voluntad [Volonté], que realiza los cambios o producciones según el principio de lo mejor" (1885). De Él manan todas las demás mónadas ${ }^{47}$ que se distinguen entre sí según su perfección, que radica, como podría esperarse, en la razón: "Y una criatura es más perfecta que otra, cuando se halla en ella algo que valga para dar razón de lo que ocurre en la otra, y es por esto por lo que se dice que actúa sobre la otra” (1885). Sin embargo, escribe Leibniz en su pretensión de superar el "dualismo epistemológico cartesiano" al panlogizar la existencia ${ }^{48}$ :

en las substancias simples no hay sino una influencia ideal de una Mónada sobre otra, la cual no puede tener su efecto más que por la intervención de Dios, en tanto que en las ideas de Dios una Mónada pide con razón, que Dios, al regular las otras desde el comienzo de las cosas, la tenga en cuenta. Porque, como una Mónada creada no puede tener una influencia física sobre el interior de otra, sólo por este medio una puede ser dependiente de otra. (Leibniz, 1977b: 51)

De todo esto Leibniz no puede concluir otra cosa más que un universo maravilloso, pero sólo uno, que no podría ser mejor: "como hay una infinidad de universos posibles en las ideas de Dios y como no puede existir sino sólo uno de ellos, es necesario que exista una razón necesaria de la elección de Dios, la cual le determine a uno antes que a otro" (Leibniz, 1977b: 53) "y esta razón no puede encon-

${ }^{46}$ Para Descartes Dios, realidad infinita, excede las posibilidades de conocimiento de una finita cosa que piensa.

47 "Sólo, pues, Dios es la Unidad Primitiva, o la substancia simple originaria, del cual son producciones todas las Mónadas creadas o derivadas; y nacen, por decirlo así, por Fulguraciones continuas de la Divinidad de momento en momento, limitadas por la receptividad de la criatura, para quien es esencial ser limitada" (Leibniz, 1977b: 47).

${ }^{48}$ Lo que también se verá que se lleva a cabo en el pensamiento de Hegel. 
trarse más que en la conveniencia o en los grados de perfección que estos mundos contienen; pues cada posible tiene derecho a pretender la existencia en la medida de la perfección que comprende" (1977b), "ésta es la causa de la Existencia del Mejor, que la Sabiduría hace conocer a Dios, su bondad le hace elegirlo y su potencia le hace producirlo" (1977b).

En este universo, escribe Leibniz presumiendo haber resuelto lógico-ontológicamente lo que Descartes había abierto con el cuidado de señalarlo epistemológicamente, ${ }^{49}$ "las almas actúan según las leyes de las causas finales, por apeticiones, fines y medios. Los cuerpos actúan según las leyes de las causas eficientes o de los movimientos. Y los dos reinos, el de las causas eficientes y el de las causas finales, son armónicos entre sî" (Leibniz, 1977b: 79). Y esto es lo que constituye el sistema de la harmonia praestabilita, el cual "hace que los cuerpos actúen como si (por imposible) no hubiera Almas; y que las Almas actúen como si no hubiera cuerpos; y que ambos actúen como si el uno influyera sobre el otro" (1977b).

Ya sólo agregará Leibniz que este desplegado de mónadas sería una ciudad de Dios (Cité de Dieu), una "Monarquía verdaderamente universal, [que] es un Mundo Moral dentro del Mundo Natural, y lo que hay más elevado y más divino en las obras de Dios; y es en él donde verdaderamente se asienta la gloria de Dios [...]" (Leibniz, 1977: 86), pues la finalidad de Dios es la felicidad de las sustancias (Leibniz, 1977a: 5). ${ }^{50}$

La felicidad de la sustancia como finalidad de la obra de Dios es la expresión de la lógica del principio de razón y manifiesta una armonía asegurada. Pero la finalidad de Dios está condicionada por la pluralidad de las sustancias expresadas por la razón respecto a lo necesariamente mejor. Además, la pluralidad de las sustancias participa de la infinitud y la perfección de acuerdo con su participación de la razón.

El infinito que para Descartes era una propiedad exclusiva de Dios, así como también la perfección, queda pretendidamente matematizado por Leibniz a través de un cálculo infinitesimal que da cuenta de todo lo que es (la pluralidad de mónadas) con necesidad racional (con razón suficiente) de las mónadas, y de cómo éstas son: aseguradamente armoniosas. Los límites con los que Descartes había construido su filosofía se transgreden en el pensamiento de Leibniz y la razón fini-

\footnotetext{
${ }^{49}$ Descartes, por ignorancia de la ley de la naturaleza en la que se conserva la misma dirección en la materia, no pudo llegar a la armonía preestablecida (Leibniz, 1977b: 80).

50 " $[\mathrm{N}]_{\mathrm{o}}$ hemos de dudar que la felicidad de los espíritus es el principal fin de Dios y que lo pone en ejecución en la medida que lo permite la armonía general”.
} 
ta del pensador de la fábula se quiere aquí infinita, aunándola con Dios, ${ }^{51}$ en un espejo, me parece, de lo que serían los alcances de la soberbia alemana protestante, ${ }^{52}$ que alcanza al menos hasta Habermas y pasa, por supuesto, por la gran cima Hegel (Dussel, 1994: 13).

Regresando a donde partimos, podemos decir, tras lo recorrido, que para Leibniz, a pesar de la pluralidad de mónadas, hay no obstante una unidad abarcante, lógica, no sustancial, que funda la realidad de una unidad sustancial que, a su vez, funda la realidad de la pluralidad de éstas por mor de lo mejor. La razón y su cálculo universal es lo que funda la realidad. En eso consistiría propiamente la ciencia:

Ese calculus es sencillamente el ser de lo ente; Leibniz concibe el ser como el calculus universalis. Ese calculus que se identifica con el ser de lo ente es llamado a veces por Leibniz natura. [...] La existencia, ella misma, es un aspecto esencial del calculus universalis mismo, en cuanto que en éste consiste el esse de todo ens y el esse de cada ens (la possibilitas de cada possible), es una cierta pretensión de existir. En el calculus se decide qué existe y qué no porque todo "qué" (todo ens) de suyo pretende existir. Así, possibilitas (esse a secas) y existentia, o "verdad de razón” y "verdad de hecho", o necesariedad y contigencia, expresan una dualidad, una tensión, inherente al esse mismo, esse que Leibniz entiende como calculus. (Martínez Marzoa, 1991: 121)

En el pensamiento de Leibniz lo científico en tanto que sería lo racional no podría ser más que una unidad de la pluralidad a pesar de la pluralidad de las sustancias (mónadas): la del principio de razón en armónica expresión de un calculus universalis que abarca todo cuanto puede ser.

51 "Descartes nos ofrece [...] una caracterización de la idea como tal, que estas dos características son en verdad la versión moderna de una dualidad que la filosofía griega había seńalado no en "el conocimiento", sino en el ser mismo, a saber: hóti éstin y ti estin: "que es" y "qué es". [...] Sin embargo, Descartes (o al menos así le parece a Leibniz) hace uso de estas nociones de "claridad" y "distinción" como si la claridad y la distinción de una percepción pudiesen ser una constatación de facto. Con esto Descartes rebaja la quaestio iuris al nivel de una cierta quaestio facti; hay en Descartes, visto por Leibniz, algo que llamaríamos "psicologismo". [...] Leibniz, por el contrario, pretende mantener la total independencia de la quaestio iuris con respecto a la quaestio facti; nada se legitima por remisión al hecho" (Martínez Marzoa, 1991: 34-35).

${ }^{52}$ Para referencias al tema, véase Paradinas Fuentes (2009) y de Juan Carlos Moreno Romo su ya referido libro La religión de Descartes. Me parece pertinente destacar aquí también las críticas que respecto al talante del pensamiento filosófico alemán hacen Arthur Schopenhauer y Friedrich Nietzsche: el primero denunciará hasta la desesperación que los alemanes quieren hacer pasar la religión nacional por la filosofía (véase "Über die Universitäts-Philosophie" incluido en el primer volumen de Parerga und Paralipomena), mientras que el segundo describirá como nacional el pensamiento de Leibniz, el de Kant y el de Hegel, exceptuando al respecto sólo el de Schopenhauer (Nietzsche, 2016: \$357). 
E. Friedrich Sauer escribe que Leibniz "no era ninguna naturaleza contemplativa, sino extremadamente activa, que se proponía realizar la armonía ahí donde el libre curso de la naturaleza todavía no lo había llevado a cabo", pues "la tendencia a la unidad puede exagerarse. Se puede ver armonía donde no la hay. [Por lo que] Leibniz exageró en su tendencia a aprehender el mundo unitariamente" (Sauer, 1973: 65). H. Glockner por su parte concluye que la importancia de Leibniz es grande, pero por otra parte su obra no es "tan enorme que no se pueda concebir la historia del espíritu humano sin ella” (Glockner, 1963: 5). Y podría ser cierto esto, sin embargo, para pronunciarse al respecto antes se tendrían que atender cuestiones acerca del "libre curso de la naturaleza" y de la "historia del espíritu humano" y la importancia filosófica de Leibniz, pero éstas escapan al ejercicio que aquí me propuse ensayar, en el que sólo he presentado el pensamiento de Leibniz como un panlogicismo en el que la metafísica del cálculo que inventa le permite imaginar una harmonia praestabilita con la que queda identificado todo lo que acontece. Más allá de esto sólo podría decir que me parece Leibniz un pensador que filosofa inspirado en la creación asegurada, más que meramente en la fabulación, como el polémico cuestionador que es Aristóteles o el metódico dubitante que es Descartes, o en la descripción atenta, como Heráclito o Schopenhauer, de mundo.

Por ello, para no llegar tan lejos como Glockner, mejor concluimos aquí con Martínez Marzoa que lo filosóficamente importante en Leibniz consiste en la elaboración de la cuestión por la verdad y no en la posesión de ella:

lo bueno está precisamente en que para Leibniz no es cuestión de principio el que vayamos a llegar alguna vez a ideas "absolutamente" o simpliciter primitivas y no meramente "tenidas por tales"; o sea: de lo que se trata no es de llegar a las nociones primitivas y de ellas a cualquier concepto, sino de investigar la naturaleza del proceso mismo; en otras palabras: no se trata de presentar el saber perfectísimo, sino de poder decir cosas acerca de cómo sería ese saber. [...] No se trata del programa de un ulterior discurso verdadero, sino de la cuestión de en qué consiste la verdad. (Martínez Marzoa, 1991: 37) 


\section{Bibliografía}

Aristóteles. (1982). TA META TA FUSIKA. METAPHYSICA. METAFÍSICA (Valentín García Yebra, Trad.). Madrid: Gredos.

Blanco Pérez, Carlos Alberto. (2005). “Leibniz y la teoría de la relación”. Thémata: Revista de Filosofía, (35), 249-258.

Caro, Hernán Darío. (2005). "Presentación a la traducción Algunas observaciones sobre el optimimo". Ideas y valores, 54(129), 47-59.

Descartes, René. (1913 [1897]). “Discours de la méthode”. En CEuvres de Descartes, IV (Charles Adam y Paul Tannery, Eds.). Paris : Librairie Philosophique Jules Vrin.

Descartes, René. (1913 [1897]). "Regulae ad directionem ingenii”. En CEuvres de Descartes, $X$ (Charles Adam y Paul Tannery, Eds.) (pp. 249-488). París: Librairie Philosophique Jules Vrin.

Descartes, René. (1972). Reglas para la dirección del espiritu en Dos opúsculos (Luis Villoro, Trad.). México: unAm.

Dussel, Enrique. (1994). 1492: el encubrimiento del otro. Hacia el origen del mito de la modernidad. La Paz: umsa; Facultad de Humanidades y Ciencias de la Educación.

ECHEVERría, Javier. (1995). "La influencia de las ideas arguesianas en el descubrimiento de Leibniz del cálculo infinitesimal”. En De Arquimedes a Leibniz. Tras los pasos del infinito matemático, teológico, físico y cosmológico [Actas del II Seminario Orotava de Historia de la Ciencia, 1992-1993] (pp. 517-536). Las Palmas de Gran Canarias: Gobierno de Canarias.

Glockner, H. (1963). Einleitung zur Monadologie. Alemania: Die Perfekte Bibliothek.

Gurwitsch, Aron. (1974). Leibniz. Philosophie des Panlogismus. Berlin: Walter de Gruyter.

Hernández Moreno, Jesús Carlos. "A propósito de mundus est fabula: Descartes y la confección de mundo”. Theoría. Revista del Colegio de Filosofía, (32), 11-30. doi: https://doi.org/10.22201/ffyl.16656415p.2017.32.435

Hernández Moreno, Jesús Carlos. (2011). "From the ego cogito, ergo sum to the World as Representation. Schopenhauer as a Reader of Descartes". En Schopenhauer-Jahrbuch, 92, 41-48.

Kant, Immanuel. (1980). Fundamentación de la metafísica de las costumbres (Manuel García Morente, Trad.). Madrid: Espasa-Calpe. 
Kant, Immanuel. (1999). Grundlegung zur Metaphysik der Sitten. Hamburgo: Meiner.

Leibniz, Gottfried Wilhelm. (1880). "Discours de la métaphysique”. En Die philosophische Schriften, Vol. 4 (Carl Immanuel Gerhard, Ed.). Berlin: Weidmann.

Leibniz, Gottfried Wilhelm. (1885). "Monadologie”. En Die philosophische Schriften, Vol. 6 (Carl Immanuel Gerhardt, Ed.) (pp. 607-623). Berlin: Weidmann.

Leibniz, Gottfried Wilhelm. (1890). "De modo distiguendi phaenomena realia ab imaginariis", "Philosophische Abhandlungen". En Die philosophische Schriften, Vol. 7 (Carl Immanuel Gerhard, Ed.). Berlin: Weidmann.

Leibniz, Gottfried Wilhelm. (1890). “Leibniz fünftes Schreiben”. En Die philosophische Schriften, Vol. 7 (Carl Immanuel Gerhard, Ed.) (pp. 389-420). Berlin: Weidmann.

Leibniz, Gottfried Wilhelm. (1977). "Discurso de metafísica". En Discurso de Metafísica. Sistema de la naturaleza. Nuevo Tratado Sobre el Entendimiento Humano. Monadología. Principios sobre la Naturaleza y la Gracia (Patricio de Azcárate, Trad.) (pp. 461-480). México: Porrúa.

Leibniz, Gottfried Wilhelm. (1977). "Monadología”. En Discurso de Metafísica. Sistema de la naturaleza. Nuevo Tratado Sobre el Entendimiento Humano. Monadología. Principios sobre la Naturaleza y la Gracia (Manuel García Morente, Trad.) (pp. 461-480). México: Porrúa.

Martínez MarzoA, Felipe. (1991). Cálculo y ser (Aproximación a Leibniz). Madrid: La balsa de la Medusa.

Mosse, George L. (1990). Die Geschichte des Rassismus in Europa. Frankfurt am Main: Fischer Taschenbuch Verlag.

Nietzsche, Friedrich. (2016). Die fröhliche Wissenschaft. Alemania: Hofenberg Sonderausgabe

Ortega y Gasset, José. (1962). La idea de principio en Leibniz y la evolución de la teoria deductiva, Obras completas, VIII, (1958-1959). Madrid: Revista de Occidente.

Paradinas Fuentes, Jesús Luis. (2009). "Leibniz y la religión”. Thémata. Revista de filosofía, (42), 123-135.

Platón. (2000). Diálogos V: Parménides, Teeteto, Sofista, Politico (Ma. I. Santa Cruz, Á. Vallejo Campos, N. Luis Cordero, Trads.). Madrid: Gredos.

SAAme, Otto. (1987). El principio de razón en Leibniz. Un elemento constitutivo de la unidad de su filosofía (Norberto Smilg y Juan A. Nicolás, Trads.). Barcelona: Laia.

SAuer, E. Friedrich,. (1973). Filósofos alemanes (María Martínez Peñaloza, Trad.). México: FCE. 
Schopenhauer, Arthur. (1986). Die Welt als Wille und Vorstellung, V. I (Sämtliche Werke I). Stuttgart/Frankfurt del Meno: Suhrkamp.

Schopenhauer, Arthur. (1986). Die Welt als Wille und Vorstellung, V. II (Sämtliche

Werke II). Stuttgart/Frankfurt del Meno: Suhrkamp.

Schopenhauer, Arthur. (2003). El mundo como voluntad y representación, V. I

(Roberto R. Aramayo, Trad.). Barcelona: Círculo de Lectores, S.A.; Fondo de Cultura Económica de Espańa, S.L.

Schopenhauer, Arthur. (2003). El mundo como voluntad y representación, V. II

(Roberto R. Aramayo, Trad.). Barcelona: Círculo de Lectores, S.A.; Fondo de Cultura Económica de España, S.L.

Schopenhauer, Arthur. (2003). El mundo como voluntad y representación, V. II

(Pilar López de Santa María, Trad.). Madrid: Trotta.

Schopenhauer, Arthur. (2004). El mundo como voluntad y representación, V. I

(Pilar López de Santa María, Trad.). Madrid: Trotta.

Unamuno, Miguel de. (1947). El espejo de la muerte. Buenos Aires: Espasa; Calpe. 